Nouveaux cahiers de la recherche en éducation

\title{
Hommage à la professeure Suzanne Pouliot
}

\section{Olivier Dezutter}

Volume 11, numéro 2, 2008

URI : https://id.erudit.org/iderudit/1017493ar

DOI : https://doi.org/10.7202/1017493ar

Aller au sommaire du numéro

Éditeur(s)

Faculté d'éducation, Université de Sherbrooke

ISSN

1911-8805 (numérique)

Découvrir la revue

Citer ce document

Dezutter, O. (2008). Hommage à la professeure Suzanne Pouliot. Nouveaux cahiers de la recherche en éducation, 11(2), 97-98.

https://doi.org/10.7202/1017493ar d'utilisation que vous pouvez consulter en ligne.

https://apropos.erudit.org/fr/usagers/politique-dutilisation/ 


\section{Hommage à la professeure Suzanne Pouliot}

La parution de ce numéro thématique des Nouveaux c@hiers de la recherche consacré à la place et au traitement de l'histoire et de la science en littérature pour l'enfance et la jeunesse, codirigé par Virginie Martel et Jean-François Boutin et qui résulte d'un colloque organisé dans le cadre du congrès de l'ACFAS en mai 2008, coïncide avec une circonstance particulière dans l'actualité de la revue et de la Faculté d'éducation de l'Université de Sherbrooke, à savoir le départ à la retraite de notre collègue Suzanne Pouliot, professeure au Département d'enseignement préscolaire et primaire. Cette circonstance nous a incités à lui dédier le présent numéro en guise de reconnaissance pour sa forte implication et sa contribution précieuse et originale dans le développement des recherches sur les littératures d'enfance et de jeunesse, dans la diffusion de cette littérature auprès des enseignants du préscolaire et du primaire, ainsi que pour son travail déterminé et efficace de soutien à la publication scientifique en français dans le contexte québécois.

Durant plus de vingt-cinq années passées au sein de notre institution, Suzanne Pouliot a jumelé activités d'enseignement et de recherche dans un champ dont elle a cherché à mieux connaitre le fonctionnement interne et externe, que ce soit du point de vue de l'analyse des textes comme en témoigne par exemple son étude de l'œuvre de Michèle Marineau ${ }^{1}$ ou du point de vue du contexte lié au fonctionnement de l'institution littéraire, engagée en ce sens, au sein du Groupe de recherche sur l'édition littéraire au Québec et de la Chaire de recherche du Canada en histoire du livre et de l'édition, dans un travail érudit de défrichage des origines et de l'évolution de la littérature pour enfants et adolescents éditée au Québec ${ }^{2}$. Sa contribution à ce numéro s'inscrit d'ailleurs dans cette ligne; elle y dresse un panorama inédit de la production théâtrale à destination des jeunes adolescents québécois durant près de deux siècles, revisitant à l'occasion une partie essentielle de l'histoire de l'éducation et de l'histoire du Canada français et du Québec. Sur un autre plan, notre collègue n'a eu de cesse d'explorer les tenants et aboutissants d'une didactique de ce qu'il est convenu d'appeler aujourd'hui la lecture littéraire auprès de tout jeunes enfants lecteurs, faisant entre autres la promotion d'un contact très précoce avec la lecture et l'objet livre ${ }^{3}$.

Disposant d'une fine connaissance du monde de l'édition, Suzanne Pouliot s'est aussi distinguée par son engagement constant en faveur de l'édition scientifique en français au sein du milieu universitaire au Québec et de la diffusion des travaux de recherche en éducation. Cet engagement

1 Pouliot, S. et Lacroix, J. (2005). Michèle Marineau. Ottawa: Les Éditions David.

2 Voir, entre autres, Pouliot, S. (2004). Les collections pour la jeunesse. In J. Michon (dir.), Histoire de l'édition littéraire au Québec au XXe siècle. Tome 2 - Le temps des éditeurs 1940-1959 (p. 185-233). Montréal: Fides.

3 Pouliot, S. et Lacroix, J. (2001). Les bébé-livres ou l'émergence de l'écrit. Sherbrooke: Éditions du CRP. 
indéfectible l'a conduite à faire partie du groupe qui a initié, au sein de la Faculté, en 1994, la création des Cahiers de la recherche en éducation, "ancêtres» de nos Nouveaux c@hiers. Depuis la création de la revue initiale, Suzanne a siégé sans discontinuité au sein du comité de direction de la revue et fortement contribué à la relance des Cahiers sous leur forme actuelle, persuadée que la parution électronique représente de nos jours une des voies d'avenir de l'édition scientifique. À l'heure où Suzanne a choisi de prendre une retraite bien méritée, le comité de direction de la revue tient à la remercier pour son extraordinaire fidélité et à l'assurer que l'héritage qu'elle nous laisse est précieux et sera l'objet de toutes nos attentions afin de continuer à servir au mieux le développement et la diffusion de la recherche en éducation.

Olivier Dezutter

Professeur titulaire Vice-doyen à la recherche et au développement international Faculté d'éducation, Université de Sherbrooke 